\title{
Horticulture and Dementia: A Review of Literature as it Affects the Domains
}

\author{
Paige Dagenhard-Trainer*, Alexandra Bryan, Abbigale Crilley, Esmeralda Diaz Quiroz, and \\ Emily Owens
}

Western Carolina University, Cullowhee, North Carolina, USA

\begin{abstract}
Dementia is a neurocognitive condition that affects individuals in the later stages of adulthood. Cognitive decline affecting memory, thinking, and behavior, alongside symptoms of depression and anxiety are typically present in individuals with dementia. Horticultural therapy is the interaction between people and plant-based activities, allowing for positive benefits through aspects of physical, social, cognitive and behavioral domains. Based on conducted research, these domains are especially impacted when horticultural therapy is utilized by older adults with dementia. Articles used within the research were based on horticulture as a therapeutic intervention for older adults and were published within a ten-year frame (2010-2020). All domains researched in relation to horticultural therapy as a modality exhibited positive benefits for this population. The cognitive domain improves the quality of life of older adults with dementia through relaxation, psychological and physiological aspects of horticulture therapy. Furthermore, the social domain is affected by decreasing social isolation, increasing positive interactions with peers, and increasing quality of life. Additionally, the behavioral domain is influenced by implementing activities such as art and music to stimulate an individual's five senses. Regarding the physical domain, activities can result in neurotic responses which are shown to improve memory. The domains of an individual with dementia can be directly affected by utilizing horticulture therapy. There are few limitations that affected this research such as resources, finances, and accessibility. This intervention is still being tested due to it being a newer form of treatment, which requires further research.
\end{abstract}

\section{Background}

Dementia is a neurocognitive condition in which there is a decline in cognitive abilities [1]. Currently, there are approximately 47 million individuals across the globe that has dementia, and there are over nine million new cases worldwide each year [2]. This disorder typically emerges during the later stages of older adulthood, but it can also begin earlier. Dementia typically presents with a decline in memory, thinking, and behavior, and is often accompanied by anxiety and depression. According to Yuan, et al. dementia tends to be a condition that requires a high volume of care (2020). Poor care given to an individual with dementia can lower their quality of life and result in early institutionalization [3]. Due to the severe impact dementia has on individuals and their caregivers, many people are admitted to long-term care facilities [2]. The transition to these facilities can be difficult, as individuals are displaced from familiar routines and their typical home environments. It is imperative that long-term care facilities implement therapeutic programming that allows the residents to experience individualized leisure to ensure a smoother transition [2].

Dementia can inhibit an individual from being able to perform daily living activities. Not being able to make decisions for themselves, explain their symptoms, or express their pre- vious medical history are all potential difficulties a client with dementia might encounter [4]. Therefore, the most common methods of treatment for dementia are nonpharmacological, which relate more to environmental adaptations and psychosocial interventions [1]. According to Noone and Jenkins, promoting the acknowledgment and expression of oneself is beneficial for individuals living with dementia [5]. Dementia is considered the disease that erodes a person's sense of self, which creates a feeling of shame or lack of self-worth. Gardening can be a way to promote positive physical and social environmental interactions [5]. Gardening is a way to not only experience the environment around the individual, but to also allow for exercise and positive leisure [6].

*Corresponding author: Paige Dagenhard-Trainer, Ph.D, LRT, CTRS, Assistant Professor, Certified Heart Math Trainer, Western Carolina University, 3971 Little Savannah Rd. 1 University Drive Cullowhee, NC, 28723-9646, USA, Tel: 828-227-2788

Accepted: May 29, 2021

Published online: May 31, 2021

Citation: Trainer PD, Bryan A, Crilley A, et al. (2021) Horticulture and Dementia: A Review of Literature as it Affects the Domains. J Hortic Sci Res 4(1):146-149 
Horticultural therapy is the interaction of people in plant-based activities and gardening by a trained horticultural therapist in order to attain specific treatment goals [7]. This type of programming provides a unique form of therapy for a wide range of different populations. This practice is beneficial for individuals involved in physical rehabilitation, as well as in vocational and community settings. Furthermore, the techniques used in this practice assist in different areas such as, but not limited to, improving memory, cognitive abilities, task initiation, language skills, and socialization. Within regards to physical rehabilitation, skills gained can include strengthening muscles, improving balance, coordination, and endurance. Additionally, vocational skills related to horticulture focus on helping individuals learn to follow directions, complete independent work, and solve problems on their own [8].

Horticultural therapy is one aspect of treatment that can lead to the achievement of therapeutic goals for older adults with dementia and is commonly used as an intervention that exposes clients to nature and outdoor leisure. This intervention, like many others, has the potential to improve one's physical, intellectual, or emotional state [1]. Gardening can be an effective form of therapy for a variety of individuals, no matter their age or diagnoses [1]. Consequently, horticulture as a therapeutic modality is important in researching in order to analyze the potential it has for improving quality of life for older adults with dementia. Because horticultural therapy can be used to improve and assist in areas such as cognitive abilities, it is vital to assess these capabilities before treatment. This programming must further aid older adults with dementia whose cognitive abilities may be declining due to this neurological disorder. Since "there are approximately 35.6 million people in the world living with dementia, and the number is doubling every 20 years" [1], the need to find ways to minimize or delay symptoms is crucial primarily due to the lack of a cure available. Implementation of horticultural therapy can be beneficial in reducing symptoms in older adults with dementia.

"The role of horticultural therapy in promoting well-being in community-dwelling people with dementia," explains that the focus of dementia research has shifted to finding ways of enabling individuals with dementia through participation in activities, including art, music, and sports [9]. Alongside this, researchers are also focusing on the use of nature and outdoor activities as a way of improving the quality of life for individuals with dementia. The article describes horticultural therapy as "a treatment modality that uses plants and plant products to improve the social, cognitive, physical, psychological, and general health and well-being of its participants" [9]. The general benefits of horticulture therapy are an efficient solution to alleviating social seclusion, as well as improving the well-being of individuals with dementia. Thus, researchers are investigating the positive relationship between horticulture therapy and dementia. This paper aims to synthesize the outcomes of horticulture therapy in relation to behavioral, social, cognitive, and physical domains for older adults with dementia.

\section{Methods}

The methodology focused on both horticultural therapy and dementia separately to gain further knowledge regarding each topic. Alongside individualized research, the topics were explored collectively to identify a research gap between the two subjects. After obtaining a general knowledge about horticultural therapy and dementia, researchers were able to compare the needs of dementia, the outcomes of horticultural therapy, and current treatment methods that are currently implemented. Furthermore, databases used for the search were EBSCOHost, USA.gov and PubMed. Search keywords used were "Horticulture journal, Horticulture therapy, Dementia, leisure, older adults, gardening, and older adults with Dementia journals". All articles selected for research were literature reviews.

Inclusion criteria were articles published within a ten-year span (2010-2020). Studies chosen were based around using horticulture or gardening as a form of treatment or therapy for older individuals diagnosed with dementia. Selected studies delved into detailed explanations of what dementia is, the symptoms related to it, and treatments. Articles that defined horticulture therapy and the benefits involved using this modality as a form of treatment were also preferred. More importantly, studies and articles that revealed results related to using horticulture as a modality for older adults with dementia were utilized. Exclusion criteria were articles published before the ten-year span starting from 2010. If the articles did not have the key words "Horticulture Therapy" and "Dementia", they were not considered reliable information to use for the research. This allows for credible research to be implemented in order to provide the most up to date results based on this intervention.

\section{Results}

As the older adult population continues to increase, there are millions of new cases of dementia each year. With this increase in numbers, more individuals are being admitted into long-term care facilities [2]. According to Yuan, et al. dementia tends to be a condition that requires a high volume of care (2020). Poor care given to an individual with dementia can lower their quality of life and even result in early institutionalization [3]. Although there are many benefits that can come from a long-term care facility, there can also be negative outcomes related to an individual's health status after admission [1]. Entering a new living environment can aggravate the symptoms of dementia because of the unfamiliarity. With this information in mind, there are four crucial domains that horticulture therapy can benefit, including behavioral, social, cognitive, and physical.

\section{Behavioral}

It is imperative that individuals with dementia, specifically those living in unfamiliar places, should be involved in leisure activities regularly in order to reduce their symptoms. Symptoms of dementia, such as disorientation, can be limited through implementing routines and familiarity [1]. A study conducted by Kathryn Mitchell explains that horticulture therapy can decrease negative symptoms such as one's feel- 
ings of depression, anger, and anxiety [2]. Moreover, research demonstrates that sensory gardens provide older adults with dementia the opportunity to stimulate their touch, taste, sight, smell, and hearing. When individuals with dementia are in the sensory garden and they are engaging, the journal article 'The Impact of a Sensory Garden for People with Dementia', states, "for people with dementia, horticulture activities are shown to improve overall well-being, increase engagement in activities, decrease disruptive behaviors, and increase positive affect" [1]. This evidence presents extensive space for growth and self-efficacy.

Additionally, in an article entitled, "The role of horticultural therapy in promoting well-being in community-dwelling people with dementia," it is explained that the focus of dementia research has shifted to finding ways of enabling individuals with dementia through participation in activities, including art, music, and sports [9]. Alongside this, researchers are also focusing on the use of nature and outdoor activities as a way of improving the quality of life for individuals with dementia. By implementing interventions constructed around the outdoors and nature, individuals an experience more positive results while doing things they enjoy. The article describes horticultural therapy as "a treatment modality that uses plants and plant products to improve the social, cognitive, physical, psychological, and general health and well-being of its participants" [9]. The article also explains that the general benefit of horticulture therapy suggests that there is an efficient solution to alleviating social seclusion, as well as improving the well-being of individuals with dementia.

\section{Cognitive}

Research demonstrates that gardening can be an effective form of therapy for a variety of individuals, no matter their age or diagnoses [1]. Consequently, horticulture as a therapeutic modality is important in researching to analyze the potential for improving quality of life for older adults with dementia. Horticultural therapy can be used to improve and assist in areas such as cognitive abilities, it is vital to assess these capabilities before treatment. This programming must further aid older adults with dementia whose cognitive abilities may be declining due to this neurological disorder. "There are approximately 35.6 million people in the world living with dementia, and the number is doubling every 20 years" [1]. Due to this, the need to find ways to minimize or delay symptoms is crucial primarily due to the lack of a cure available.

Accordingly, the sensory garden provides the older adults with dementia to stimulate their touch, taste, sight, smell, and hearing. Evidence from Cheng, et al. study concludes that "older adults indicated that relaxation, psychological, and the physiological aspects of gardening were the most satisfying" [6]. Cheng, et al. (pg. 397) states, "the predictors for survival among older adults found that gardening was associated with a lowered risk of mortality as it promoted a sense of self-efficacy that helped older adults to reduce the adverse effects of psychological stress" (2010). Other factors according to the study, 'Identifying the Satisfaction Derived from Leisure Gardening by Older Adults' associated to gardening are it lowered "risk of mortality as it promoted a sense of self-efficacy that helped older adults to reduce the adverse effects of psychological stress" [6].

Likewise, a study in Seoul, South Korea, gardening for older adults in their late seventies and early eighties revealed a potential benefit for increased cognitive function. Six activities within the intervention were completed by the older adults, including cleaning a garden plot, digging, fertilizing, raking, planting/transplanting, and watering. Before and after the activity sessions, blood was drawn two times from each individual in order to assess levels of "brain nerve growth factors, including brain-derived neurotrophic factor (BDNF), vascular endothelial growth factor (VEGF) and platelet derived growth factor (PDGF)," [10]. The levels of these growth factors were notably increased after the gardening interventions, uncovering a possible benefit of gardening and horticultural activities in older adults cognitive functioning [10].

\section{Physical}

Within regards to physical rehabilitation, skills gained can include strengthening muscles, improving balance, coordination, and endurance [8]. Horticulture therapy can help them physically by increasing functional independence through participation in physical activity programs with community garden projects [9]. The sensory garden provides the older adults with dementia to stimulate their touch, taste, sight, smell, and hearing. When individuals are in the sensory garden and they are engaging, according to the journal article 'The Impact of a Sensory Garden for People with Dementia', the authors stated that, "For people with dementia, horticulture activities are shown to improve overall well-being, increase engagement in activities, decrease disruptive behaviors, and increase positive affect" [1].

In the same study as mentioned above, 'Benefits of Gardening Activities for Cognitive Function According to Measurements of Brain Nerve Growth Factor Levels', a 20-minute gardening activity with low-to-moderate physical activity intervention led to the increase of BDNF. BDNF is in the neurotrophin family and supports neural survival. The increase of BDNF, which is a key protein, promoted memory cell increase and growth in the hippocampus and cortical. Patients with dementia had increased levels of brain nerve growth by the end of the study [10]. Other factors according to the study, 'Identifying the Satisfaction Derived from Leisure Gardening by Older Adults' associated to gardening are it lowered "risk of mortality as it promoted a sense of self-efficacy that helped older adults to reduce the adverse effects of psychological stress" [6]. Horticulture therapy helps older adults with dementia believe in their own ability to do something independently. It gives them something to release their worries and stress on. While participants garden, their minds are occupied, focused, and concentrated on what is in front of them for the time being.

\section{Social}

Studies show that horticulture is a popular leisure interest amongst older adults across the world. Not only is it a common interest of older adults, but it has also been proven to be beneficial in improving the overall life satisfaction of many 
[6]. There are many benefits to horticulture and according to the article, "Being Grounded": Benefits of Gardening for Older Adults in Low-Income Housing,' "therapeutic benefits of horticultural therapy include lessening an individual's sense of loss and providing opportunities for creativity, self-expression, social interaction, and sensory stimulation; improving self-esteem; practicing fine and gross motor skills; and improving eye-hand coordination" [11].

Similarly, a literature review of six studies explains that a variety of different horticultural therapy interventions were implemented with older adults, some of these interventions included gardening, cooking with herbs, sensory stimulation, and more. These older adults were diagnosed with dementia and their ages averaged at 80 years. Group sessions ranged from thirty minutes to an hour, one to three times a week, for six to ten weeks. The findings in these six studies revealed that the individuals had positive interactions with their peers, had improved moods, increased their abilities to reminisce, and increased their quality of life [12].

Furthermore, several studies in a systematic review of nature environment-based interventions for individuals with neurological disabilities, including dementia, revealed an impressive amount of research related to gardening and older adults with dementia. Natural environments aid significantly in positive aspects of psychosocial health results, including emotional and social health for persons with dementia. Access to green space and natural environments have been consistent with residents in different settings to experience less stress, have improved mental and emotional health, along with an increase is positively socializing with peers and an improved quality of life [13].

\section{Discussion}

Throughout the results, four domains were addressed: Behavioral, cognitive, physical, and social. Each domain was addressed as it applies to the use of horticultural therapy for older adults with dementia. Researchers discovered that this intervention consistently shows positive results in relation to behavioral health, cognitive skills, physical health, and social skills. Horticulture has been studied on multiple occasions and has been proven to be successful for older adults. It is imperative that these studies are continued in order to improve the health of older adults, specifically those with dementia.

Limitations within this research include finding sources that discuss horticulture therapy and how it affects the domains amongst individuals with dementia. Horticulture therapy has limited research so discovering articles that correlated it to the four domains was difficult. In addition, there may be potential difficulties amongst individuals acquiring both the resources and money needed to implement this intervention. Another factor to consider is that the sources utilized throughout this paper were based in places outside of the United States which can cause an influence in specific aspects. Potential speculations involve the focus on cognitive function over other areas such as physical skills, behavioral health, and social skills. Alongside this, there is a prevalence of research for older adults with dementia over other popu-

\section{lations.}

In essence, our findings have further solidified the numerous benefits that horticultural therapy holds regarding older adults with dementia. Through using horticulture as a modality with this population, literature has exhibited various beneficial improvements within the domains. The domains include behavioral, physical, social, and cognitive skills. The research conducted contributes to the literature and continues to exemplify the potential and capability that horticultural therapy has to offer for older adults with dementia.

Future research does need to be completed in order to advance our knowledge on the benefits and possible drawbacks that are presented regarding horticultural therapy for older adults diagnosed with dementia. Allowing for an expansion of geographical locations in relation to experimental research may diversify results and expand our knowledge on the benefits of horticultural therapy. Experimental studies must be completed in order to close research gaps and further aid older adults with dementia.

\section{References}

1. Collins H, Van Puymbroeck M, Hawkins BL, et al. (2020) The impact of a sensory garden for people with dementia. Therapeutic Recreation Journal 54: 48-63.

2. Mitchell K, Van Puymbroeck M (2019) Recreational therapy for dementia-related symptoms in a long-term care setting: A case study. Therapeutic Recreation Journal 53: 165-174.

3. Yuan $Q$, Tan TH, Wang P, et al. (2020) Staging dementia based on caregiver reported patient symptoms: Implications from a latent class analysis. PLoS One 15: e0227857.

4. Watkins S, Murphy F, Kennedy C, et al. (2020) Caring for older people with dementia in the emergency department. $\mathrm{Br} \mathrm{J}$ Nurs 29: 692-699.

5. Noone S, Jenkins N (2018) Digging for Dementia: Exploring the experience of community gardening from the perspectives of people with dementia. Aging Ment Health 22: 881-888.

6. Cheng E HP, Patterson I, Packer J, et al. (2010) Identifying the satisfactions derived from leisure gardening by older adults. Annals of Leisure Research 13: 395-419.

7. American Horticultural Therapy Association [AHTA] (2012) Definitions and positions.

8. Williams S (2020) About horticulture therapy.

9. Noone S, Innes A, Kelly F, et al. (2017) "The nourishing soil of the soul": The role of horticultural therapy in promoting well-being in community-dwelling people with dementia. Dementia 16: 897-910.

10. Park SA, Lee AY, Park HG, et al. (2019) Benefits of gardening activities for cognitive function according to measurement of brain nerve growth factor levels. Int J Environ Res Public Health 16: 760.

11. Wang D, Glicksman A (2013) "Being grounded": Benefits of gardening for older adults in low-income housing. Journal of Housing for the Elderly 27: 89-104.

12. Watts C, Pei-Chun Hsieh (2015) The use of horticulture-based programs to promote engagement for older adults with dementia. Therapeutic Recreation Journal 49: 257-260.

13. Lakhani A, Norwood M, Watling DP, et al. (2019) Using the natural environment to address the psychosocial impact of neurological disability: A systematic review. Health Place 55: 188-201. 